\title{
KLOE-2 Results on Hadron Physics
}

\section{Paolo GAUZZI *}

Dipartimento di Fisica, Sapienza Università di Roma e INFN Sezione di Roma, P.le A.Moro 2, 00185 Rome (Italy)

E-mail: Paolo.Gauzzieromal.infn.it

The most recent results from the KLOE experiment on hadron physics are presented. The measurement of the running of the fine-structure constant $\alpha_{e m}$ in the time-like region below $1 \mathrm{GeV}$ is described. The new measurement of Dalitz plot of $\eta \rightarrow \pi^{+} \pi^{-} \pi^{0}$ is presented and its relation with the determination of the light quark masses is discussed. The measurements of the Transition Form Factors of $\phi \rightarrow \eta e^{+} e^{-}$and $\phi \rightarrow \pi^{0} e^{+} e^{-}$are also reported.

The KLOE-2 Collaboration will take data until mid 2018 aiming to collect $5 \mathrm{fb}^{-1}$ increasing the data set, in order to produce new precision measurements in hadron phyics.

XVII International Conference on Hadron Spectroscopy and Structure - Hadron2017

25-29 September, 2017

University of Salamanca, Salamanca, Spain

\section{${ }^{*}$ on behalf of the KLOE-2 Collaboration}




\section{Introduction}

From 2001 to $2006 \mathrm{KLOE}$ has collected $2.5 \mathrm{fb}^{-1}$ of data at the peak of the $\phi(1020)$, plus 250 $\mathrm{pb}^{-1}$ off-peak at the $e^{+} e^{-}$collider DAФNE[1] in Frascati. In 2008 a new interaction scheme of the DAФNE $\phi$-factory has been adopted, to increase the machine luminosity. In November 2014 a new data-taking campaign, referred to as KLOE-2 experiment[2] has started. Until September 2017 about $4 \mathrm{fb}^{-1}$ have been collected. The DAФNE peak luminosity has been $2 \times 10^{32} \mathrm{~cm}^{-2} \mathrm{~s}^{-1}$, allowing KLOE-2 to collect about $10 \mathrm{pb}^{-1}$ per day. By the end of data-taking, foreseen for mid 2018, the goal of $5 \mathrm{fb}^{-1}$ should be reached, doubling the statistics of the first KLOE data-taking.

The KLOE-2 detector has been upgraded with the installation of: $(i)$ an Inner Tracker close to the DAФNE Interaction Point (IP), made of four layers of cylindrical GEMs, to improve the track and vertex reconstruction; (ii) a tagging system for scattered electrons in $\gamma \gamma$ processes, consisting of two different detectors, a High Energy Tagger (HET: scintillator hodoscopes readout by PMTs) placed after the first bending magnet of the machine, and a Low Energy Tagger (LET: LYSO clystal calorimeters readout by $\mathrm{SiPM}$ ) placed at about $1 \mathrm{~m}$ from the IP; (iii) new small angle calorimeters, the QCALT (tungsten and scintillator tiles, readout by SiPM) as instrumentation of the correcting quadrupoles inside the detector, and the CCALT (LYSO crystals, readout by SiPM), very close to the IP to increase the acceptance for low polar angle particles.

In this paper recent results on hadron physics from the analysis of the KLOE data set are reported.

\section{Running of $\alpha_{e m}$}

It is well known that the fine-structure constant is a running parameter due to vacuum polarization effects, $\alpha_{e m}\left(q^{2}\right)=\frac{\alpha_{e m}(0)}{1-\Delta \alpha}$. The correction $\Delta \alpha$ is the sum of the lepton, the five ligthest quarks, and the top quark contributions: $\Delta \alpha\left(q^{2}\right)=\Delta \alpha_{l e p}\left(q^{2}\right)+\Delta \alpha_{\text {had }}^{(5)}\left(q^{2}\right)+\Delta \alpha_{\text {top }}\left(q^{2}\right)$. The top quark contribution is negligible at low energies, the leptonic part is calculated with very high precision in QED, while the hadronic part cannot be calculated with perturbative methods. It can be evaluated from experimental data by means of the dispersion relation $\left(s=q^{2}\right)$, $\Delta \alpha_{\text {had }}^{(5)}(s)=-\frac{\alpha(0) s}{3 \pi} \int_{s_{0}}^{\infty} \frac{R_{\text {had }}\left(s^{\prime}\right)}{s^{\prime}\left(s^{\prime}-s-i \varepsilon\right)} d s^{\prime}$, where $R_{\text {had }}(s)=\frac{\sigma\left(e^{+} e^{-} \rightarrow \text { hadrons }\right)}{\sigma\left(e^{+} e^{-} \rightarrow \mu^{+} \mu^{-}\right)}$. The value of $\alpha_{e m}(s)$ in the time-like region can be extracted from the ratio of the differential cross-secion of $e^{+} e^{-} \rightarrow \mu^{+} \mu^{-} \gamma$, with the photon from Initial State Radiation (ISR), and the corresponding cross-section obtained from the Monte Carlo (MC) simulation with $\alpha_{e m}(s)=\alpha_{e m}(0)$ [3].

$$
\left|\frac{\alpha_{e m}(s)}{\alpha_{e m}(0)}\right|^{2}=\frac{\left.d \sigma_{\text {data }}\left(e^{+} e^{-} \rightarrow \mu^{+} \mu^{-} \gamma(\gamma)\right)\right|_{I S R} / d \sqrt{s}}{\left.d \sigma_{M C}^{0}\left(e^{+} e^{-} \rightarrow \mu^{+} \mu^{-} \gamma(\gamma)\right)\right|_{I S R} / d \sqrt{s}}
$$

Events with two tracks with opposite charge at polar angle greater than $50^{\circ}$ are selected. The photon is required to be at low polar angle, less than $15^{\circ}$, then is not detected and its momentum is reconstructed from the kinematics of the event. In a sample of $1.7 \mathrm{fb}^{-1}$, about $4.5 \times 10^{6} \mu^{+} \mu^{-} \gamma$ events have been selected. In figure 1 left an example of separation of the signal from the main background, $\pi^{+} \pi^{-} \gamma$ events, is shown together with the applied cut. The residual background is less than $1 \%$. In figure 1 right is shown the ratio of eq.(2.1) compared to the theoretical predictions[4]. In the time-like region of $q^{2}, \Delta \alpha$ is a complex quantity, and the real part of it can be expressed as:

$$
\operatorname{Re} \Delta \alpha=1-\sqrt{\left|\alpha_{e m}(0) / \alpha_{e m}(s)\right|^{2}-(\operatorname{Im} \Delta \alpha)^{2}}
$$



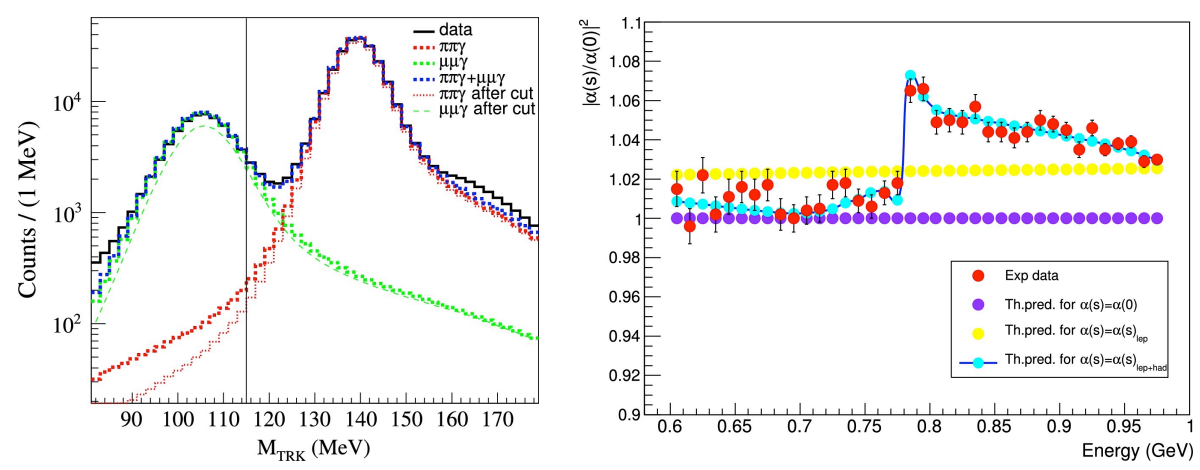

Figure 1: Left: $\mu^{+} \mu^{-} \gamma$ and $\pi^{+} \pi^{-} \gamma$ separation, ( $M_{T R K}$ is the mass of the reconstructed charged track); right: $\left|\frac{\alpha_{e m}(s)}{\alpha_{e m}(0)}\right|^{2}$ as a function of the dimuon invariant mass, compared with the theoretical predictions[4]).

From the optical theorem follows that $\operatorname{Im} \Delta \alpha=-\frac{\alpha_{\text {em }}}{3} R_{\text {had }}(s)$, and in figure 2 left is reported the behaviour of $\operatorname{Im} \Delta \alpha$ obtained from the KLOE data on the $2 \pi$ cross-section[5] that is the dominant contribution in this energy range. The real part from eq.(2.2) is shown in figure 2 right.
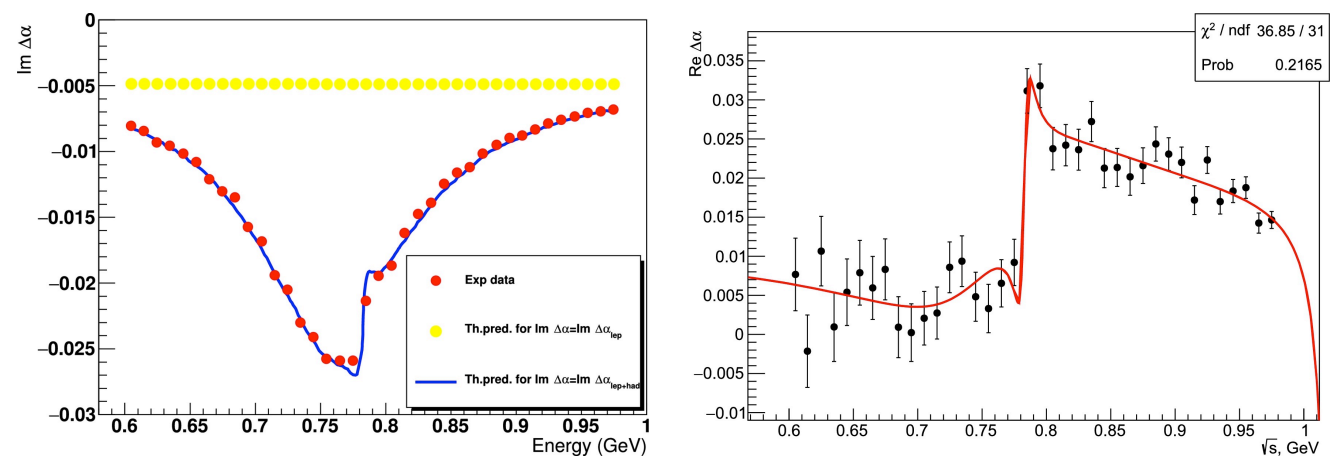

Figure 2: Left: $\operatorname{Im} \Delta \alpha$ from $\operatorname{KLOE} \pi^{+} \pi^{-} \gamma$ cross section (red points) and from a compilation of other measurements (blue curve); right: $\operatorname{Re} \Delta \alpha$ from eq. (2.2), the red line is the fit described in the text.

The superimposed curve is a fit performed by parametrizing the $\omega(782)$ and $\phi(1020)$ as simple Breit-Wigner and describing the $\rho(770)$ with the Gounaris-Sakurai parametrization, and adding a non resonant term. The free parameters are listed in table 1 , while the mass and the width of the $\phi$, the $\omega$ width as well as the product $\operatorname{Br}\left(\phi \rightarrow e^{+} e^{-}\right) \operatorname{Br}\left(\phi \rightarrow \mu^{+} \mu^{-}\right)$have been fixed to the PDG values. Assuming lepton universality and correcting for phase space, $\operatorname{Br}\left(\omega \rightarrow \mu^{+} \mu^{-}\right)=$ $(6.6 \pm 1.4 \pm 1.7) \times 10^{-5}$ is obtained (PDG: $\left.\operatorname{Br}\left(\omega \rightarrow \mu^{+} \mu^{-}\right)=(9.0 \pm 3.1) \times 10^{-5}\right)$.

3. $\eta \rightarrow \pi^{+} \pi^{-} \pi^{0}$

The $\eta \rightarrow 3 \pi$ decay is a strong process, isospin violating, being the electromagnetic contribution strongly suppressed according to the Sutherland theorem. This decay proceeds through a Lagrangian term proportional to the $d-u$ quark mass difference. The decay amplitude is proportional to $Q^{-2}$, the quadratic quark mass ratio is defined as $Q^{2}=\frac{m_{s}^{2}-\hat{m}^{2}}{m_{d}^{2}-m_{u}^{2}}$ with $\hat{m}=\frac{1}{2}\left(m_{u}+m_{d}\right) . Q=24.3$ 


\begin{tabular}{|c|c|c|c|}
\hline Parameter & Fit without $\rho-\omega$ interf. & Fit with $\rho-\omega$ interf. & PDG \\
\hline$M_{\rho}(\mathrm{MeV})$ & $775 \pm 6$ & $778 \pm 7$ & $775.26 \pm 0.25$ \\
$\Gamma_{\rho}(\mathrm{MeV})$ & $146 \pm 9$ & $147 \pm 10$ & $147 \pm 0.9$ \\
$M_{\omega}(\mathrm{MeV})$ & $782.7 \pm 1.1$ & $783.4 \pm 0.8$ & $782.65 \pm 0.12$ \\
$\operatorname{Br}\left(\omega \rightarrow \mu^{+} \mu^{-}\right) \operatorname{Br}\left(\omega \rightarrow e^{+} e^{-}\right)$ & $(4.3 \pm 1.8) \times 10^{-9}$ & $(4.4 \pm 1.8) \times 10^{-9}$ & $(6.5 \pm 2.3) \times 10^{-9}$ \\
$\chi^{2} / \mathrm{ndf}$ & 1.19 & 1.15 & \\
\hline
\end{tabular}

Table 1: Result of the fit to $\operatorname{Re} \Delta \alpha$.

can be obtained from a combination of pion and kaon masses[6]. With this value, the decay width can be calculated at different orders of ChPT, $\Gamma_{L O}\left(\eta \rightarrow \pi^{+} \pi^{-} \pi^{0}\right)=66 \mathrm{eV}, \Gamma_{N L O}\left(\eta \rightarrow \pi^{+} \pi^{-} \pi^{0}\right)=$ 160-210 eV, and $\Gamma_{N N L O}\left(\eta \rightarrow \pi^{+} \pi^{-} \pi^{0}\right)=230-270 \mathrm{eV}$, approaching very slowly the experimental value (PDG) $\Gamma\left(\eta \rightarrow \pi^{+} \pi^{-} \pi^{0}\right)=(300 \pm 12) \mathrm{eV}$.

The measurement of the Dalitz plot of $\eta \rightarrow \pi^{+} \pi^{-} \pi^{0}$ can provide stringent constraints on the light quark masses. The Dalitz plot distribution is expressed in terms of the normalized variables: $X=\sqrt{3} \frac{T_{+}-T_{-}}{Q_{\eta}}, Y=3 \frac{T_{0}}{Q_{\eta}}$ where the $T_{i}$ are the kinetic energies of the pions and $Q_{\eta}=$ $m_{\eta}-2 m_{\pi^{ \pm}}-m_{\pi^{0}}$. The squared amplitude is usually parametrized as a Taylor expansion around the center, $|A(X, Y)|^{2}=N\left(1+a Y+b Y^{2}+c X+d X^{2}+e X Y+f Y^{3}+g X^{2} Y+\ldots\right)$. The Dalitz plot has been recently measured by KLOE with the decay $e^{+} e^{-} \rightarrow \phi \rightarrow \eta \gamma$, with $\eta \rightarrow \pi^{+} \pi^{-} \pi^{0}$, on a sample of $1.6 \mathrm{fb}^{-1}$ of data, corresponding to $4.7 \times 10^{6}$ events[7], improving the previous measurement [8]. In figure 3 the Dalitz plot is shown, and in table 2 the parameters from the fit are listed.
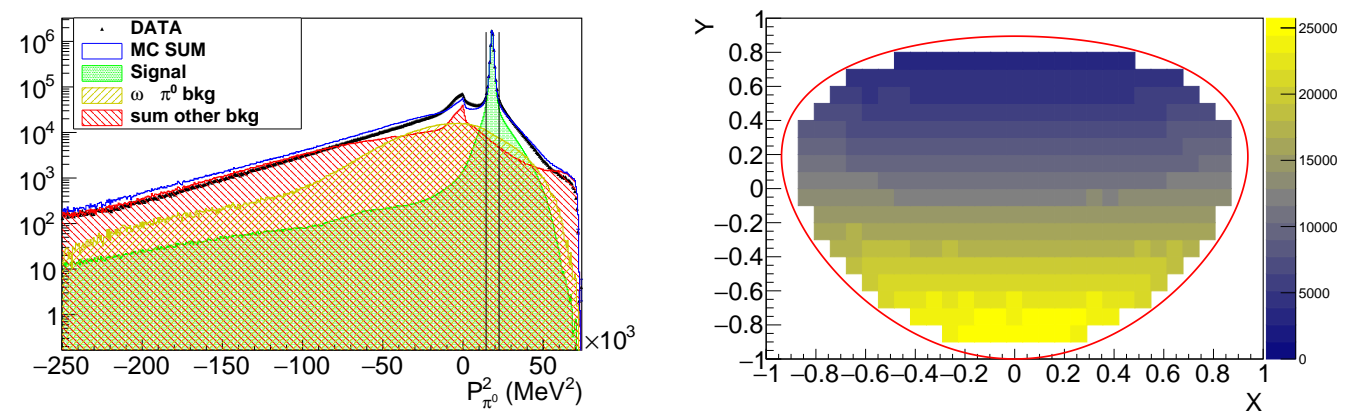

Figure 3: Left: Missing mass with respect to $\eta \pi^{+} \pi^{-}$, the selected region is the narrow band around the peak; right: Dalitz plot of $\eta \rightarrow \pi^{+} \pi^{-} \pi^{0}$.

\begin{tabular}{|c|c|c|c|c|c|}
\hline & $a$ & $b$ & $d$ & $f$ & $g$ \\
\hline KLOE ('16)[7] & $-1.095 \pm 0.004$ & $0.145 \pm 0.006$ & $0.081 \pm 0.007$ & $0.141 \pm 0.011$ & $-0.044 \pm 0.016$ \\
KLOE ('16)[7] & $-1.104 \pm 0.004$ & $0.142 \pm 0.006$ & $0.073 \pm 0.005$ & $0.154 \pm 0.008$ & \\
KLOE ('08)[8] & $-1.090 \pm 0.020$ & $0.124 \pm 0.012$ & $0.057 \pm 0.017$ & $0.14 \pm 0.02$ & \\
WASA[9] & $-1.144 \pm 0.018$ & $0.219 \pm 0.051$ & $0.086 \pm 0.023$ & $0.115 \pm 0.037$ & \\
BESIII[10] & $-0.128 \pm 0.017$ & $0.153 \pm 0.017$ & $0.085 \pm 0.018$ & $0.173 \pm 0.035$ & \\
\hline
\end{tabular}

Table 2: $\eta \rightarrow \pi^{+} \pi^{-} \pi^{0}$ Dalitz plot parameters of $\operatorname{KLOE}(' 16)$ compared to previous measurements.

The $c$ and $e$ parameters are $\mathrm{C}$-violating and are compatible with zero. At present the most recent KLOE measurement is the only one sensitive to the $g$ parameter.

Recently, methods based on dispersion relations have been setup to constrain the light quark mass 
ratios, by making use of fits to the experimental Dalitz plots. In one of the most recent papers on this subject Colangelo and collaborators[11] fit the KLOE Dalitz plot of $\eta \rightarrow \pi^{+} \pi^{-} \pi^{0}$ to obtain $Q=22.0 \pm 0.7$, and using the ratio $\frac{m_{s}}{\hat{m}}=27.30 \pm 0.34$ from Lattice QCD the authors determined the $u / d$ quark mass ratio $\frac{m_{u}}{m_{d}}=0.44 \pm 0.03$.

\section{Transition Form Factors}

The Transition Form Factors (TFFs) describe the coupling of mesons to photons and provide information about the nature of the mesons and their structure. Recently the interest in the TFFs has been renewed due to the discrepancy of more than three standard deviation between the theoretical and the experimental value of the anomalous magnetic moment of the muon. The main contribution to the hadronic part of the muon anomaly is the vacuum polarization, but the second one in order of relevance comes from the hadronic Light-by-Light (HLbL) scattering. The leading contribution to the HLbL is the single pseudoscalar exchange, in which the TFFs appear at the two vertices of the meson propagator. The calculation of this contribution is model dependent since the exchanged meson is off-shell, and the TFFs for off-shell meson are not measurable quantities. Nevertheless any experimental information the TFFs, both for space-like and time-like $q^{2}$, can help in constraining the models used in the calculations[12]. The TFFs for time-like $q^{2}$ can be studied by means of the Dalitz decays $P \rightarrow V \ell^{+} \ell^{-}$or $V \rightarrow P \ell^{+} \ell^{-}$, as functions of the invariant mass $q^{2}=m^{2}\left(\ell^{+} \ell^{-}\right)$. According to Vector Meson Dominance (VMD) the TFFs are usually parametrized as $F\left(q^{2}\right)=$ $1 /\left(1-\frac{q^{2}}{\Lambda^{2}}\right)$, where $\Lambda$ is a characteristic mass parameter. For almost all these decays the measured slopes, $\Lambda^{-2}$, are in agreement with VMD predictions except for the process $\omega \rightarrow \pi^{0} \mu^{+} \mu^{-}$. KLOE studied the process $\phi \rightarrow \eta e^{+} e^{-}$with $\eta \rightarrow 3 \pi^{0}$, by selecting events with two charged tracks and six prompt photons, and obtained the $\operatorname{Br}\left(\phi \rightarrow \eta e^{+} e^{-}\right)=(1.075 \pm 0.007 \pm 0.038) \times 10^{-4}[13]$. From the fit to the $e^{+} e^{-}$invariant mass distribution obtained the TFF shown in figure 4 left. The slope is $\Lambda^{-2}=(1.28 \pm 0.10 \pm 0.09) \mathrm{GeV}^{-2}$ compatible with VMD prediction $\Lambda_{V M D}^{-2} \simeq 1 \mathrm{GeV}^{-2}$. KLOE also measured $\phi \rightarrow \pi^{0} e^{+} e^{-}$, by selecting events with two tracks and two prompt photons, and obtained $\operatorname{Br}\left(\phi \rightarrow \pi^{0} e^{+} e^{-}\right)=\left(1.35 \pm 0.05_{-0.10}^{+0.05}\right) \times 10^{-5}$. The slope is $\Lambda^{-2}=(2.02 \pm 0.11)$ $\mathrm{GeV}^{-2}[14]$, which does not agree with VMD expectations (figure 4 right).
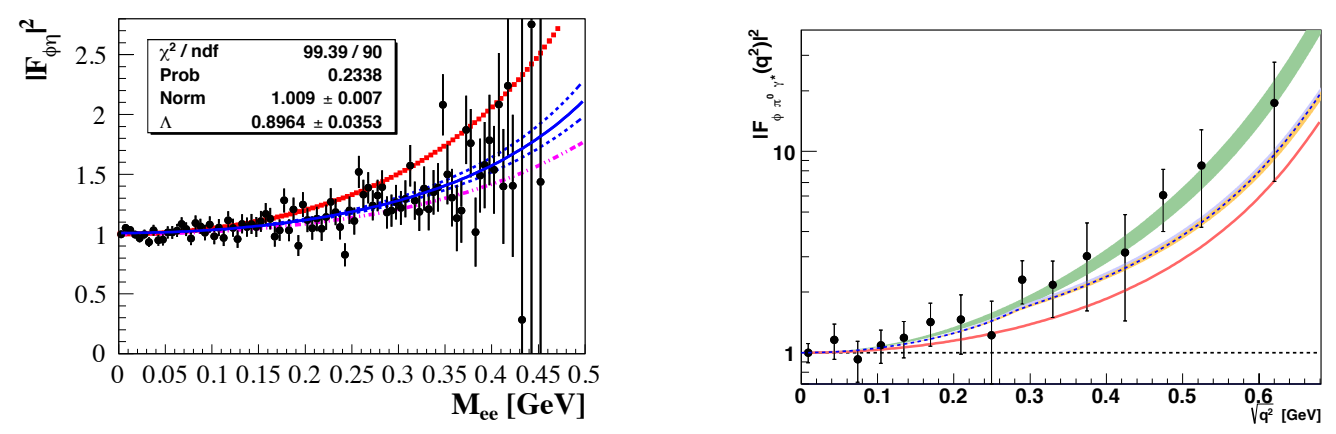

Figure 4: TFFs: left - $\phi \rightarrow \eta e^{+} e^{-}$, the blue line is the VMD prediction[13]; right - $\phi \rightarrow \pi^{0} e^{+} e^{-}$, the red line is the VMD prediction[14]. 


\section{Conclusions}

The KLOE-2 Collaboration is continuing to exploit the high statistics data samples collected during the first period of KLOE data-taking, to perform precision measurements in hadron physics. The precision study of the light meson properties will continue with increased statistics, with the data of the KLOE-2 campaign, that will end in 2018 with the luminosity goal of $5 \mathrm{fb}^{-1}$. Main topics of the future studies will be: TFFs, improving $\phi \rightarrow \eta e^{+} e^{-}, \pi^{0} e^{+} e^{-}$and measuring $\phi \rightarrow$ $\eta \mu^{+} \mu^{-}, \eta \pi^{+} \pi^{-}$and also $e^{+} e^{-} \rightarrow \pi^{0} \gamma \gamma_{I S R}$; the rare $\eta$ decays $\left(\eta \rightarrow \pi^{0} \gamma \gamma, \eta \rightarrow 4\right.$ charged tracks, limits on $\eta \rightarrow \gamma \gamma \gamma, \pi^{+} \pi^{-}$); the $\gamma \gamma$ process $e^{+} e^{-} \rightarrow e^{+} e^{-} \pi^{0}$. Also the searches of dark bosons belonging to a secluded sector will be performed, improving the existing limits and looking for new hypothetical particles.

\section{References}

[1] A. Gallo et al., DAFNE status report, Conf. Proc. C060626 (2006) 604-606.

[2] G. Amelino-Camelia et al., Physics with the KLOE-2 experiment at the upgraded DA $\$$ NE, Eur. Phys. J. C68 (2010) 619-681, [1 003 . 3868].

[3] KLOE-2 collaboration, A. Anastasi et al., Measurement of the running of the fine structure constant below $1 \mathrm{GeV}$ with the KLOE Detector, Phys. Lett. B767 (2017) 485-492, [1609. 06631].

[4] F. Jegerlehner, Electroweak effective couplings for future precision experiments, Nuovo Cim. C034S1 (2011) 31-40, [1107.4683].

[5] KLOE collaboration, D. Babusci et al., Precision measurement of $\sigma\left(e^{+} e^{-} \rightarrow \pi^{+} \pi^{-} \gamma\right) / \sigma\left(e^{+} e^{-} \rightarrow \mu^{+} \mu^{-} \gamma\right)$ and determination of the $\pi^{+} \pi^{-}$contribution to the muon anomaly with the KLOE detector, Phys. Lett. B720 (2013) 336-343, [1212.4524].

[6] R. F. Dashen, Chiral SU(3) x SU(3) as a symmetry of the strong interactions, Phys. Rev. 183 (1969) $1245-1260$.

[7] KLOE-2 collaboration, A. Anastasi et al., Precision measurement of the $\eta \rightarrow \pi^{+} \pi^{-} \pi^{0}$ Dalitz plot distribution with the KLOE detector, JHEP 05 (2016) 019, [1601.06985].

[8] KLOE collaboration, F. Ambrosino et al., Determination of $\eta \rightarrow \pi^{+} \pi^{-} \pi^{0}$ Dalitz plot slopes and asymmetries with the KLOE detector, JHEP 05 (2008) 006, [0801.2642].

[9] WAS A-AT-COSY collaboration, P. Adlarson et al., Measurement of the $\eta \rightarrow \pi^{+} \pi^{-} \pi^{0}$ Dalitz plot distribution, Phys. Rev. C90 (2014) 045207, [1 406 .2505].

[10] BESIII collaboration, M. Ablikim et al., Measurement of the Matrix Elements for the Decays $\eta \rightarrow \pi^{+} \pi^{-} \pi^{0}$ and $\eta / \eta^{\prime} \rightarrow \pi^{0} \pi^{0} \pi^{0}$, Phys. Rev. D92 (2015) 012014, [1506.05360].

[11] G. Colangelo, S. Lanz, H. Leutwyler and E. Passemar, $\eta \rightarrow 3 \pi$ : Study of the Dalitz plot and extraction of the quark mass ratio Q, Phys. Rev. Lett. 118 (2017) 022001, [1610 . 03494].

[12] F. Jegerlehner and A. Nyffeler, The Muon g-2, Phys. Rept. 477 (2009) 1-110, [0 902 . 3360].

[13] KLOE-2 collaboration, D. Babusci et al., Study of the Dalitz decay $\phi \rightarrow \eta e^{+} e^{-}$with the KLOE detector, Phys. Lett. B742 (2015) 1-6, [1 409.4582$]$.

[14] KLOE-2 collaboration, A. Anastasi et al., Measurement of the $\phi \rightarrow \pi^{0} e^{+} e^{-}$transition form factor with the KLOE detector, Phys. Lett. B757 (2016) 362-367, [1601.06565]. 